\title{
Límites de la tarificación vial ${ }^{1}$
}

Louis de Grange. Universidad Diego Portales, Santiago, Chile.

Rodrigo Troncoso. Universidad del Desarrollo, Santiago, Chile.

RESUMEN | Para muchos académicos y profesionales de la ingeniería de transporte, el concepto de tarificación por congestión se ha transformado en el paradigma de la eficiencia en el uso de la escasa infraestructura vial y del desincentivo al uso excesivo del automóvil. Si bien la tarificación vial puede ser, en varios casos, una buena herramienta de gestión de tráfico, comporta también una serie de riesgos y sobreexpectativas que es importante tener en consideración a la hora de diseñar, implementar y administrar un eficiente esquema de tarificación vial.

En este trabajo presentamos una serie de antecedentes, basados muchos de ellos en la literatura especializada y en evidencia empírica, que permiten describir los riesgos potenciales y eventuales sobreexpectativas que enfrenta una política de tarificación por congestión, lo que puede ser de gran ayuda para impulsar una correcta implementación de esta política de gestión de tráfico.

PALABRAS ClAVE $\mid$ tarificación vial, riesgos, eficiencia, bienestar social, efectos distributivos.

ABSTRACT | For many academic and professional transportation engineers, the concept of congestion pricing has become the paradigm of efficiency regarding the use of scarce road infrastructure and implementing dissuasive measures to deter excessive car use. While road pricing can be considered, in many cases, as a good traffic management tool, it also involves a number of risks and overexpectations that are important to consider when designing, implementing and managing an efficient road pricing scheme.

This work presents a series of records, many of them based on specialized literature and empirical evidence, that help to describe the potential risks and possible overexpectations that can come into play in a congestion pricing policy. Such information can be of great help in order to promote the correct implementation of traffic management policy.

KEYWORDS | urban road pricing, risks, efficiency, social well-being, distributive effects.

Recibido el 11 de abril de 2013, aprobado el 9 de septiembre de 2013

E-mail: louis.degrange@udp.cl | rtroncoso@udd.cl

Correspondencia autor principal: Universidad Diego Portales, Facultad de Ingeniería, Vergara 432, 8370190, Santiago, Chile.

1 Parte de este trabajo fue presentado en el xvi Congreso Chileno de Ingeniería de Transporte, celebrado en Santiago entre el 21 y el 25 de octubre de 2013. 


\section{Introducción}

La tarificación vial por congestión ha sido una herramienta de gestión de tráfico ampliamente aprobada por economistas, ingenieros de transporte y técnicos en general que se vinculan profesionalmente con la planificación urbana y de sistemas de transporte. Sin embargo, existen motivos teóricos y evidencia empírica que destacan los potenciales efectos adversos de este tipo de política pública para regular el uso excesivo del automóvil. En este trabajo ofrecemos una revisión de antecedentes relevantes sobre los eventuales efectos contraproducentes de la tarificación vial por congestión, y que representan componentes de riesgo para el diseño, implementación y difusión de esta política pública.

En este contexto, Charles Lave (1995) ya advertía a mediados de la década de los noventa sobre la complejidad del problema:

Ha sido práctica común entre los economistas del transporte colocar en la pizarra el diagrama convencional [de la teoría de la congestión], señalar el evidente carácter óptimo de sus soluciones en materia de precios, para luego sentarse a esperar que el mundo adopte esta solución obviamente correcta. Bien, ya hemos estado esperando durante setenta años, y vale la pena preguntarse qué aspectos del problema se nos han estado escapando. ¿Por qué el mundo es renuente a hacer lo obvio? (p. 465; traducción propia $)^{2}$.

Los argumentos teóricos a favor de la tarificación vial se sustentan en el principio microeconómico de que aquellos bienes cuya producción o consumo genera externalidades negativas tienden a ser sobreproducidos (o sobreconsumidos), y que una manera eficiente de lograr una producción o consumo socialmente óptimo sería la implementación de impuestos pigouvianos.

Lo, Hickman y Walstad (1996) destacan, entre las ventajas de la tarificación vial, que esta permite: (i) internalizar los costos de las externalidades negativas del uso excesivo del automóvil; (ii) captar ingresos que pueden ser asignados a proyectos de transporte (público o privado); y (iii) recolectar información que puede ser usada en la gestión del tráfico y en el diseño de nuevas políticas de transporte.

Sin embargo, estos conceptos no implican que cualquier esquema de tarificación vial se traduzca, necesariamente, en mayores beneficios sociales. Levinson (2010) plantea que la mayoría de los análisis cuantitativos relacionados con los impactos de la tarificación vial se han basado en el uso de modelos teóricos y simulaciones computacionales sobre sus efectos esperados, y no en análisis empíricos y seguimientos antes-después en los lugares donde se ha implementado esta medida.

En un sistema con imperfecciones en el mercado del trabajo (e.g. impuesto a la renta) y con economías de aglomeración, los efectos de la congestión y los eventuales beneficios de una tarificación vial no van a ser capturados correctamente por

2 "It has been a commonplace event for transportation economists to put the conventional [congestion theory] diagram on the board, note the self-evident optimality of pricing solutions, and then sit down waiting for the world to adopt this obviously correct solution. Well, we have been waiting for seventy years now, and it's worth asking what are the facets of the problem we have been missing. Why is the world reluctant to do the obvious?" (p. 465). 
un análisis aislado del mercado del transporte, como los que se utilizan normalmente (Parry \& Bento, 2001). En general, no se suele considerar otros mercados que se puedan ver afectados negativamente por la tarificación vial (análisis de equilibrio general en lugar de equilibrio parcial), tampoco los efectos redistributivos que puede generar tal medida (ganadores, perdedores y su proporción), ni la aceptación que ella pueda tener entre los distintos agentes que componen la sociedad (compensaciones).

En la siguiente sección reportamos una revisión bibliográfica, considerando el contexto y objetivo del presente artículo. En la tercera sección describimos, sobre la base de la revisión bibliográfica realizada, algunos de los riesgos que comporta un esquema de tarificación vial y que debieran tenerse presente. En la cuarta sección sintetizamos algunos aspectos relevantes que deben considerarse al momento de impulsar, diseñar e implementar una política de tarificación vial. Finalmente, se presentan las principales conclusiones de este trabajo.

\section{Antecedentes bibliográficos}

Existe abundante literatura a favor de implementar la tarificación vial por congestión para desincentivar un uso excesivo del automóvil, y también amplio consenso técnico en torno a ella como positiva herramienta de gestión de la demanda en redes viales congestionadas. Después del trabajo pionero de Pigou (1920), autores como Walters (1961) y Vickrey (1963) sentaron las bases para la fijación de un impuesto por el uso de la infraestructura de acuerdo con los costos de congestión. Más recientemente, esta idea ha vuelto a cobrar fuerza en múltiples trabajos, tanto teóricos como empíricos (véase, entre otros, Smith, 1979; Newbery, 1990; Hau, 1992, 1998, 2005; Small, 1992; Small \& Gómez-Ibáñez, 1998; Nash \& Sansom, 2001; De Borger \& Proost, 2001; De Palma \& Lindsey, 2011; Anas \& Lindsey, 2011; Basso \& Jara-Díaz, 2012). Una amplia revisión del estado del arte se presenta en Lindsey (2006) y en Tsekeris y Voß (2009).

Sin embargo, a pesar de la amplia justificación teórica, en muy pocas ciudades se han implementado políticas de precios que contemplen la internalización de los costos externos por el uso de infraestructura. Las razones incluyen problemas asociados a los efectos redistributivos entre los distintos grupos afectados y a los bajos beneficios esperados que esta política tendría, en comparación con los altos costos políticos de implementarla.

Los análisis de costos y beneficios asociados a la tarificación vial normalmente se concentran en el mercado del transporte. Un análisis más completo requiere del uso de modelos de equilibrio general que contemplen la interacción con otros mercados, como el mercado laboral; la existencia de otros mercados con distorsiones, o la heterogeneidad de los agentes afectados. Según Arnott, De Palma y Lindsey (1993, 1998), el modelo estándar de congestión es pobre y contiene ambigüedades, pero estas limitaciones se pueden superar usando modelos estructurales. 


\section{Efectos redistributivos y costos políticos}

Para implementar exitosamente un sistema de tarificación vial es fundamental la comunicación con la ciudadanía y el énfasis que se haga en los beneficios de esta medida. Sobre la viabilidad política de la tarificación vial, Ison (1998) discute aspectos relacionados con su implementación, para concluir que la equidad y el destino de los recursos recaudados por la medida son quizá los aspectos más importantes de tener en cuenta a fin de que no sea considerada como inaceptable por la mayor parte de la sociedad.

Levinson (2010) argumenta que la aceptabilidad política de la tarificación vial por congestión depende fuertemente de la distribución (y percepción de la distribución) de las ganacias y pérdidas que esta medida genera sobre los distintos grupos afectados (positiva y negativamente). Mohring y Harwitz (1962) plantean que, bajo ciertas condiciones, lo recaudado por tarificación vial alcanza para cubrir los costos de inversión, oportunidad del capital, y operación y mantenimiento de un sistema vial. Luego, la tarificación vial funcionaría como un precio que permite asignar recursos de manera óptima sin distorsionar ningún otro mercado. Sin embargo, este postulado ha sido fuertemente cuestionado por muchos otros autores, como se expone en esta sección.

King, Manville y Shoup (2007) plantean que a pesar de que planificadores de transporte y economistas puedan estar de acuerdo en cuanto a la eficiencia económica de la tarificación vial y su capacidad para reducir la congestión, es riesgosa política y posiblemente desastrosa. No es pertinente asumir que las personas se vayan a mostrar a favor de dicha medida solo por ser económicamente eficiente. Por ejemplo, Goodwin (1997) argumenta que discutir el tema de la tarificación vial sin una atención explícita a la utilización de los recursos económicos obtenidos dificulta enormemente el soporte político y aceptación que ella pueda obtener.

En una revisión de la literatura sobre los efectos redistributivos de la tarificación vial, Levinson (2010) destaca que tanto los trabajos teóricos como los empíricos ofrecen resultados mixtos en esta materia. Foster $(1974,1975)$ fue de los primeros en argumentar que la tarificación vial discrimina en contra de los automovilistas más pobres y a favor de los más ricos. No obstante, se debe tener presente que se trata de un contexto de automovilistas. Los individuos realmente pobres no usan el automóvil de manera habitual, sino el transporte público, y en muchos casos la tarificación vial puede mejorar los niveles de servicio de los buses en superficie.

Arnott, De Palma y Lindsey (1994) encuentran que una tarifa que elimine las colas va a tender a ser regresiva, porque beneficia a los que más valoran el tiempo, que a su vez suelen tener mayores ingresos. Pero que la introducción de un descuento fijo podría revertir esta situación, ya que representaría un beneficio proporcionalmente mayor para usuarios de menor ingreso. Glazer y Niskanen (2000) consideran dos modos congestionados, uno lento y uno rápido. Concluyen que la tarificación del modo congestionado rápido (autopista urbana) podría aumentar el bienestar agregado y sería progresiva. En el plano empírico, Kulash (1974), Segal y Stein-

meier (1980) y Small (1983) encuentran que la tarificación vial probablemente tenga efectos regresivos. 
Usando modelos dinámicos de congestión con viajeros heterogéneos, Arnott et al. (1994, 1998) comparan los efectos sobre el bienestar de diferentes grupos de ingresos, y encuentran que la tarificación vial es regresiva, y específicamente que los beneficios de esta política recaen sobre los más ricos, mientras que los costos lo hacen sobre los más pobres. Estos resultados confirman estudios anteriores (Layard, 1977; Cohen, 1987; Evans, 1992) que, usando modelos estáticos de congestión, también concluyen que la tarificación vial es regresiva. En un estudio más reciente, Raux y Souche (2004) confirman este resultado.

Preocupados por los grupos en riesgo, Bonsall y Kelly (2005) concluyen que la tarificación vial aumenta la exclusión social para los automovilistas de menos recursos y los viajeros cautivos del automóvil. Franklin (2005) estima la variación del índice de Gini asociada a la implementación de una política de tarificación vial sobre un puente congestionado que tiene como alternativa un servicio de buses. Si bien la tarifa produce un aumento en el bienestar social total, este bienestar se distribuye de manera inequitativa entre los viajeros, perjudicando a los más pobres y favoreciendo a los más ricos.

Parry y Bento (2001), y Safirova, Houde, Coleman, Harrington y Lipman (2006) consideran los efectos de la tarificación vial sobre el mercado laboral, distorsionado por impuestos al trabajo. Ambos grupos de autores advierten que el aumento generalizado del costo de viaje producto de la tarificación vial desincentiva el trabajo, exacerbando las pérdidas preexistentes de los impuestos a la renta. Estas pérdidas pueden ser mayores a las ganancias de eficiencia pigouviana por internalizar los costos de la congestión.

En un trabajo reciente, Anderstig, Berglund, Eliasson, Andersson y Pyddoke (2012) concluyen que la tarificación vial podría tener un efecto negativo sobre los ingresos de las personas, mayor a la ganancia de eficiencia del mercado del transporte. Sin embargo, Parry y Bento (2001) agregan que si los ingresos monetarios de la tarificación por congestión se utilizan para reducir los impuestos al trabajo, restringiendo así la distorsión en ese mercado, se podría producir una ganancia adicional de eficiencia del mismo orden de magnitud que la obtenida por la internalización de la congestión. Concluyen también que el reciclaje de los ingresos por tarificación vial hacia subsidios al transporte público produce un impacto positivo, pero menor, sobre la oferta laboral. Asimismo, Peters y Kramer (2012) argumentan que si los ingresos obtenidos de una política de tarificación vial por congestión se utilizan para compensar cargas tributarias en el mercado laboral o para mejorar los servicios de transporte público que sean alternativa para los automovilistas, se puede obtener una importante ganancia neta de los beneficios sociales.

Algunos trabajos incorporan en el análisis las externalidades positivas por aglomeración que se perderían con la tarificación vial. Graham y Van Dender (2011) plantean que la existencia de economías de aglomeración o de densidad y sus respectivos beneficios no son capturados correctamente por los clásicos enfoques costobeneficio aplicados a los mercados de transporte. Sobre la determinación de la tarificación óptima, Arnott (2007) argumenta que si se consideran las externalidades positivas por aglomeración, el impuesto socialmente óptimo por congestión debe ser menor que el que internaliza completamente las externalidades por congestión. 
El impacto de la tarificación vial sobre el valor del suelo de las zonas tarificadas y las periféricas también genera efectos redistributivos. Según Lindsey (2012), la tarificación vial aplicada en un sector céntrico de la ciudad (por ejemplo, un cordón tarificado) produce un aumento en el valor de los inmuebles dentro de la zona tarificada y en sectores cercanos. Conforme se reduce la distancia con el sector tarificado, mayor es este aumento. De manera opuesta, en los sectores más alejados del centro tarificado, los bienes inmuebles tienden a reducir su valor. Lindsey (2012) explica que en un contexto de equilibrio general, la ventaja relativa del mejor acceso a las zonas tarificadas se traduce en una plusvalía de las propiedades céntricas a cambio de una reducción en el valor de las propiedades alejadas del centro tarificado. En contraste, Glazer y Van Dender (2002) argumentan que la tarificación vial por congestión podría generar una reducción en el precio de los bienes inmuebles en las áreas afectas a la tarificación, generando con ello una fuerte oposición a esta medida. La reducción en el precio de los bienes inmuebles sería mayor incluso que los ingresos monetarios recibidos como recaudación por la tarificación. Usando un enfoque de simulaciones, Eliasson y Matsson (2001) encuentran que el efecto de la tarificación sobre la localización sería pequeño en comparación con los que tendría sobre los patrones de viaje. En general, señalan que la tarificación haría la ciudad menos dispersa, pero no por una densificación del centro tarificado, sino del anillo interior de los suburbios.

\section{Dificultades de implementación}

El efecto en bienestar de la tarificación vial depende en forma crítica de su implementación. Las limitaciones prácticas que se suelen enfrentar hacen imposible tarificar óptimamente desde un punto de vista teórico. La evidencia empírica muestra que existe una amplia diferencia entre las políticas de tarificación implementadas en la práctica y las recomendaciones proporcionadas por la teoría económica (De Borger \& Proost, 2001). Principalmente, los impuestos cobrados por tarificación vial no se diferencian en términos de tiempo o lugar, así como tampoco distinguen por tipo de vehículo.

Verhoef, Nijkamp y Rietveld (1996) muestran que las tarifas óptimas por congestión dependen de la razón relativa de los tiempos de viaje a flujo libre entre las rutas alternativas, de las capacidades de ambas rutas y de las elasticidades preciodemanda. Plantean una serie de inconvenientes operacionales y de costo para la implementación de las tarifas first-best (óptimas), razón por lo cual argumentan la necesidad de usar tarifas second-best. Sin embargo, obtienen también que los beneficios de tarificaciones second-best son normalmente una fracción muy baja de la tarifa first-best (cerca de solo un 10\%). Liu y McDonald (1999) respaldan este resultado para un proyecto en California (State Route 91 en Orange County).

Para Parry y Bento (2002), el problema es que el efecto de la tarificación vial sobre la renta agregada de las personas es siempre negativo, lo que puede generar pérdidas de bienestar mayores a los beneficios obtenidos en el mercado de transporte. Eliasson y Mattson (2006) estiman que, antes de aplicar medidas compensatorias, la tarificación vial en Estocolmo generó un costo neto de 482 coronas suecas por residente al año; pero con posterioridad a la reinversión de los fondos recaudados, 
el beneficio neto fue de 222 coronas suecas al año por residente. Por otra parte, evidencia empírica muestra que el esquema de tarificación vial de Singapur (cobro por circular en una determinada área de la ciudad) habría generado una reducción en el bienestar social, aun cuando el tráfico en los períodos punta se redujo (menor excedente de los consumidores). Wilson (1988) reportó que después de la implementación de la tarificación vial en Singapur, el tráfico en el período punta se redujo un 65\%, y los usuarios de buses aumentaron 8\%; sin embargo, un 44,1\% de los viajeros habituales en automóvil durante los períodos punta aumentaron sus tiempos de viaje, mientras que un $36,1 \%$ los redujo.

\section{Tarificación vial y contaminación}

Un argumento comúnmente usado a favor de la tarificación vial es que esta ayudaría a reducir los niveles de emisión de gases contaminantes por parte de los vehículos. Sin embargo, es importante precisar que el concepto de tarificación vial por congestión busca, como su nombre lo dice, corregir una falla de mercado específica, que es la congestión vial. Si bien la contaminación es una externalidad negativa asociada al uso del automóvil, es diferente a la congestión y, por lo tanto, debiera ser corregida con un instrumento también diferente. Por ejemplo, un impuesto a los combustibles sería una medida más eficiente que la tarificación vial para enfrentar el problema específico de la contaminación causada por los automóviles.

Al ser la congestión y la contaminación dos fallas de mercado diferentes, pero vinculadas al uso del automóvil, ambas podrían ser incorporadas simultáneamente en el diseño de los mecanismos de tarificación vial que se pretenda impulsar. Existen algunos trabajos en los que se incorpora el efecto de las emisiones en los análisis de equilibrio (de usuarios y óptimo del sistema) en redes de transporte congestionadas (Tzeng \& Chen, 1993; Rilett \& Benedek, 1994; y Benedek \& Rilett, 1998). En estos trabajos se considera la emisión de un único contaminante en el análisis de equilibrio en redes de transporte.

Tzeng y Chen (1993) formulan un problema multiobjetivo en el que incorporan, además de los tiempos de viaje, un costo adicional correspondiente a las emisiones de monóxido de carbono (co). Rilett y Benedek (1994) desarrollan una extensión del clásico problema de asignación de tráfico (Beckmann, McGuire \& Winsten, 1956), en la que especifican una función de costos medioambientales, concluyendo que existe un conflicto entre minimizar los tiempos totales de viaje en el sistema y el nivel total de emisiones.

Benedek y Rilett (1998) encuentran que al incorporar las emisiones de co en la modelación de la asignación de tráfico, ellas pueden reducirse en un 7\% respecto de una asignación óptima del sistema (basada en el segundo principio de Wardrop).

Sugawara y Niemeyer (2002) formulan una metodología basada en un modelo de asignación de viajes que busca minimizar las emisiones (modelo de emisiones óptimas, EO), considerando como contaminante al monóxido de carbono. El modelo fue comparado con los modelos de asignación de tráfico óptima de usuarios (UE) y asignación óptima del sistema (so), encontrando que el enfoque EO reduce entre 3,3\% y 5\% las emisiones respecto de los enfoques ue y so. También encuentran que el resultado varía dependiendo del nivel de congestión de la red. 
Por ejemplo, en calles locales con menores velocidades promedio (excluyendo a las autopistas urbanas, que se consideran menos congestionadas que las calles locales), la reducción en las emisiones de co del enfoque EO respecto al uE y al so varía entre un $23,9 \%$ y un $26 \%$. Sin embargo, cuando aumenta la congestión en toda la red, estos porcentajes se reducen un poco, pero siempre quedan por sobre el $7 \%$.

Ahn y Rakha (2008) consideran, dentro de la elección de ruta de los viajeros en la red de transporte, el efecto no solo en las emisiones, sino también en el consumo de energía. Utilizan especificaciones de las emisiones (y consumo eléctrico) más sofisticadas que los trabajos antes citados (co, hidrocarburos, dióxido de carbono y óxido de nitrógeno), pero confirman los resultados de Sugawara y Niemeyer (2002), y de Yin y Lawphongpanich (2006).

\section{Sobreexpectativas y riesgos de la tarificación vial}

\section{Sobreexpectativas de la tarificación vial}

Una evaluación adecuada de los beneficios que se puedan lograr tarificando determinadas vías requiere reconocer las limitaciones de tal medida, evitando una excesiva simplificación del problema. De esta manera se evitarían expectativas demasiado optimistas por parte de las autoridades y los usuarios, que pueden terminar convirtiéndose en frustración y altos costos políticos.

Safirova, Harrington, Houde y Coleman (2007) exponen algunos "mitos y conceptos erróneos" que rodean la tarificación vial por congestión. Concluyen que la tarificación vial no es siempre la solución y que es probable que las mejoras en el bienestar a las que conducen vayan "de lo modesto a lo moderado". Según estos autores, varios de los "mitos" han sido generados por investigadores, o provienen de la imaginación de observadores casuales, mientras otros son simple consecuencia de un análisis supuestamente racional, pero incorrecto. Estos "mitos", al ser ampliamente aceptados, afectan la manera en que los tomadores de decisiones piensan, diseñan y eventualmente implementan una política pública como la tarificación vial.

A continuación nos referimos a algunas expectativas y afirmaciones que suelen producirse sobre los esquemas de tarificación vial óptimos:

1. La tarificación vial elimina la congestión. Esta afirmación es falsa, no es el objetivo de la tarificación vial acabar con la congestión. En el mejor de los casos (e hipotético), la congestión solo se reduce a un nivel socialmente óptimo. Teóricamente existe un nivel óptimo de congestión, ya que de lo contrario habría excesiva oferta vial. Y mientras más valore la población usar el auto, mayor será el nivel óptimo de congestión.

2. La tarificación vial baja a la gente del auto. Esto no siempre ocurre. En muchos casos solo logra cambiar la ruta que escogen los automovilistas, que suele ser más larga que la usada cuando no había tarificación vial. Si el automovilista no tiene alternativas, simplemente pagará y percibirá la tarificación vial como un impuesto más que no genera beneficios sociales. La gente dejará el auto solo en la medida en que tenga buenas alternativas de transporte. De hecho, las ciudades con esquemas 
exitosos de tarificación vial, como Londres y Singapur, cuentan con excelentes sistemas de transporte público (De Grange, 2010).

3. La tarificación vial reduce la contaminación. No existen garantías de que la contiminación vaya a disminuir. Incluso, existe el riesgo de que ocurra justo lo contrario. Como se expone en el trabajo de Yin y Lawphongpanich (2006), si los automovilistas, para evitar las rutas tarificadas, usan rutas más largas, aumentará el kilometraje y con ello los niveles de emisión de contaminantes. En un trabajo previo, May y Milne (2000) encuentran que la tarificación vial aumenta las distancias de viaje.

4. La tarificación vial aumenta el bienestar social. Existen varios escenarios en los que la tarificación vial podría disminuir el bienestar social. Como los beneficios máximos teóricos de la tarificación vial están acotados, puede ocurrir que los costos de implementación y administración de esta medida sean demasiado altos o los efectos negativos sobre otros mercados sean muy grandes, y la sociedad en su conjunto reduzca su nivel de bienestar. Por ejemplo, varios trabajos sugieren que el efecto neto en bienestar podría depender en forma crítica del destino que se dé a los fondos recaudados por la tarificación.

5. Los costos de la congestión vial son muy elevados. Este mito proviene del incorrecto análisis de comparar la situación congestionada con la situación sin congestión. Efectivamente, puede que la congestión genere costos importantes, pero nada garantiza que la tarificación vial logre eliminarla. De hecho, un diseño de tarificación vial socialmente óptimo llevará a un nivel también óptimo de congestión. Eliminar completamente la congestión sería muy ineficiente desde la perspectiva social, básicamente porque los beneficios marginales de la medida tenderían a cero, y las personas perderían más de lo que ganan. Esta lógica es consistente con el concepto de "precio de la anarquía" (Papadimitriou, 2001; Roughgarden, 2003; Correa, Schulz \& Stier-Moses, , 2004; Yang, Xu \& Heydecker, 2010), que establece que los máximos beneficios que se obtienen de una tarificación vial socialmente óptima, respecto del equilibrio de mercado, son acotados. Por ejemplo, Basso, Guevara, Gschwender \& Fuster (2011) muestran que los beneficios de pistas exclusivas para buses pueden ser superiores a los de la tarificación, además de recaer en todos los actores.

6. La tarificación vial debe ser implementada solo en lugares de alta congestión. Si bien parece una afirmación razonable, porque estos serían los lugares con mayores beneficios potenciales de una reducción en congestión, puede ser recomendable partir con precios bajos en lugares donde se prevé que habrá congestión en un futuro próximo. De la misma forma, debido a que las redes viales son buenos "conductores" de la congestión, al tarificar una determinada área o camino puede trasladarse la congestión a sectores no tarificados que antes no presentaban problemas importantes de congestión.

7. La tarifa por congestión incentiva a los individuos a vivir más cerca de los lugares céntricos de la ciudad. Este resultado se fundamenta en los análisis de ciudades monocéntricas (un gran centro al que llegan los viajeros desde los sectores 
periféricos). Sin embargo, cuando existen múltiples centros demográficos, como normalmente ocurre en las grandes ciudades, se produce también un efecto de expansión de la ciudad, ya que algunos individuos se trasladan hacia las afueras en lugar de hacia el centro. Este comportamiento se observa normalmente en sistemas de tarificación vial basados en cordones (cordon pricing).

8. La inversión en transporte público y la tarificación vial son complementarias. Esto se justificaría por las supuestas economías de escala en el transporte público, por lo que al captar usuarios provenientes del automóvil, mejoraría el nivel de servicio del transporte público. Sin embargo, la evidencia empírica ha mostrado que esta sinergia es bastante menor que aquella pronosticada por los modelos teóricos.

9. Los ingresos por tarificación vial pueden ser usados para financiar nuevos proyectos de infraestructura vial. Normalmente, los ingresos por tarificación vial no permiten cubrir más que una fracción de nuevas obras viales. Por otro lado, existe el riesgo de agravar el efecto regresivo de la medida si se termina beneficiando exclusivamente a automovilistas de altos ingresos. Además, los proyectos de infraestructura pública socialmente más rentables no se limitan a las zonas o a los montos recaudados por la tarificación vial.

10. La tarificación vial siempre aumenta el bienestar social. Este argumento se ha basado principalmente en modelos microeconómicos teóricos demasiado sencillos. Al considerar el impacto de la tarificación vial sobre otros mercados relevantes con distorsiones o externalidades, como el mercado laboral o las economías de aglomeración, es posible que las pérdidas sociales que se producen en el otro mercado excedan los beneficios esperados de internalizar la congestión.

\section{Riesgos de la tarificación vial}

A continuación presentamos algunos riesgos potenciales que debieran considerarse al evaluar una política de tarificación vial por congestión.

Riesgo 1: Las autoridades de gobierno tienen incentivos para maximizar la recaudación de ingresos, diseñando tarifas con ese objetivo en lugar de otras que busquen maximizar el bienestar social o minimizar el costo total del sistema (Evans, 1992). Es posible demostrar que un equilibrio en el que se maximizan los ingresos por tarificación vial puede llevar a una situación que es peor desde una perspectiva social, que el equilibrio de mercado sin tarificación. Verhoef y Small (2004) también advierten del riesgo de que las autoridades se comporten como un monopolio que tiende a maximizar la recaudación en lugar de minimizar los tiempos totales de viaje.

Riesgo 2: Las autoridades tienen menores incentivos para implementar medidas operacionales de gestión de tráfico o nuevas inversiones en infraestructura, ya que si reducen la congestión, se reduce también la justificación del cobro de la tarificación vial (Evans, 1992).

Riesgo 3: La tarificación vial puede ser regresiva. Quienes dejan de usar el automóvil producto de la tarificación vial son aquellos que tienen una menor disposición a pagar por el uso de las vías, que suelen ser las personas de menores ingresos. De esta 
manera, los viajeros más ricos siguen usando el auto, mientras que los de menores ingresos dejan de usarlo. La descongestión se traduce, entonces, en una mejora en los tiempos de viaje para los más ricos, que, expresada en dinero, puede ser mayor que el monto que pagan por la tarificación vial. Mientras tanto, quienes se ven obligados a dejar de usar el automóvil necesariamente ven reducido su nivel de bienestar (Parry, 2008).

No obstante este último riesgo, la eventual regresividad puede verse compensada en zonas caracterizadas por un alto uso del transporte público de superficie, el que corresponde a usuarios de menores ingresos que se verían beneficiados por una menor congestión. El beneficio de descongestión tiene un mayor valor cuando existen vehículos de transporte público con alta tasa de ocupación.

\section{Aspectos que considerar en el diseño e implementación de una política de tarificación vial por congestión}

Para Bhatt (1993), las principales barreras para implementar esquemas de tarificación vial se relacionan con las inequidades en la distribución de los costos y beneficios que esta medida produce, por lo que el diseńo de mecanismos compensatorios es un elemento central para impulsarla. Estas inequidades pueden ser horizontales o verticales. La inequidad horizontal corresponde a las diferencias en niveles de servicio que experimentan los viajeros del sistema en los distintos sectores geográficos de la ciudad, como consecuencia de la tarificación vial. La inequidad vertical corresponde a las diferencias generadas en el presupuesto económico de los individuos de los distintos grupos afectados.

King et al. (2007) plantean que la factibilidad política de la tarificación vial depende principalmente de qué grupos se verán beneficiados con las recaudaciones obtenidas. Argumentan que cobrar por el uso de calles congestionadas genera la oportunidad de reinvertir en la misma ciudad en la que se está implementando el cobro, mejorando con ello la percepción de los usuarios del sistema.

Steg y Schuitema (2007) describen algunas condiciones en las cuales el efecto de la tarificación vial puede ser más efectivo en la reducción del uso del automóvil. La principal es la existencia de un transporte público de alta calidad (como en Londres y Singapur), de tal forma que este sea una alternativa real para el automovilista. Debido a que la demanda o disposición a pagar por usar el automóvil depende, además del costo monetario de usar el vehículo, de los costos generalizados de las otras alternativas de transporte, un buen transporte público aumenta la elasticidad del automovilista, generando con ello una mayor disposición a cambiar su comportamiento frente a la tarificación vial. Sin embargo, si el automovilista no considera el transporte público como alternativa, simplemente puede percibir la tarificación vial como un impuesto adicional que no genera beneficios claros, con la consecuente reprobación de esta medida. Por lo tanto, un buen transporte público pareciera ser una precondición para que la tarificación vial sea viable.

Richards (1992) propone ocho aspectos relevantes de considerar para el diseño e implementación de una correcta política de tarificación vial: 
1. Impacto sobre la demanda: debe preverse los efectos directos e indirectos sobre el comportamiento de los automovilistas afectos a la tarificación vial. Esto implica anticipar potenciales cambios de ruta, de destino de viaje, de horario o incluso de modo de transporte.

2. Impacto sobre la oferta: debe preverse cómo cambian los niveles de oferta tanto en el sector tarificado como en los restantes sectores, horarios y modos de transporte, a fin de estimar si es necesario efectuar ajustes de ofertas.

3. Impacto sobre la economía urbana: debe estimarse el efecto sobre el comercio detallista, sectores financieros y de servicios, y también sobre los bienes inmuebles al interior del sector tarificado, tanto en el corto como en el largo plazo.

4. Análisis de equidad: implica determinar cuáles son los grupos afectados por la medida (positiva y negativamente), así como la magnitud del impacto, mediante, por ejemplo, un análisis costo-beneficio desagregado por grupo de individuos.

5. Medioambiente y seguridad: se requiere estimar el impacto que la medida propuesta puede generar sobre los niveles de emisiones y contaminación en general, así como sobre la tasa de accidentabilidad.

6. Viabilidad financiera: se debe determinar cuáles son los costos de implementar y explotar la medida, y compararlos con los ingresos generados. Además, deben incluirse los eventuales cambios en los ingresos monetarios de los agentes involucrados; por ejemplo, la recaudación en transporte público, las ventas de combustibles y su respectivo efecto en la recaudación de impuestos.

7. Capacidad de fiscalización y cumplimiento: se debe determinar qué tan efectivo es el método de ejecución de la política propuesta, y cuál es su nivel de cumplimiento o morosidad de pago.

8. Tecnología: debe especificarse qué plataforma tecnológica debe implementarse para gestionar los cobros y su recaudación, y para facilitar los procesos de cobranza y de fiscalización.

Además, para hacer ciudadanamente aceptable una política de tarificación vial, se requiere:

- Identificar claramente los problemas existentes; estos deben ser visibles, y no haber sido resueltos con otras medidas tomadas anteriormente (e.g. restricción vehicular).

- Transmitir los beneficios de la medida tanto al inicio de su aplicación como en el tiempo, mostrando los resultados esperados y alcanzados.

- Esperar el momento oportuno. Es muy difícil convencer a los votantes de pagar por un plan antes de que hayan visto sus beneficios. Sin embargo, el apoyo tiende a aumentar después de que la política opera en estado de régimen. Esto permite recomendar un aumento paulatino y progresivo de la política en el tiempo, incorporando así nuevas zonas o calles tarificadas, nuevos períodos, o eventuales aumentos de tarifas. 
- Lograr que la medida sea percibida como justa por parte de los usuarios del sistema de transporte. Esto implica proteger o compensar expresamente a los grupos más vulnerables que puedan verse afectados por ella. De hecho, cuando en Singapur fue implementada la tarificación vial, se la acompañó de una reduccción del impuesto a la compra de automóviles (el que seguía siendo elevado).

- Definir con anticipación en qué se reinvertirán los fondos recaudados. En Londres, los ingresos van al sistema de autobuses, mientras que en Oslo se utilizan para construir más carreteras. Una falta de definición respecto del uso de los recursos obtenidos genera desconfianza por parte de los distintos grupos de interés, ya que no basta con indicar que se redujo la congestión. En Londres, la introducción de cientos de buses nuevos tuvo un impacto visible importante, previo a la puesta en marcha de la tarificación vial.

- Conseguir apoyo de líderes de opinión. Esto implica que no es suficiente contar con el respaldo de técnicos en la materia, sino que, además, se requiere sumar a líderes políticos, ambientales, económicos, etcétera, para lograr una mayor confianza en esta medida.

Sin embargo, antes de implementar la medida, debe mejorarse y optimizarse la gestión de tráfico en los sectores congestionados mediante nuevas tecnologías de información y sistemas de control vehicular dinámico para vehículos y peatones. De la misma forma, es importante que se regule el uso de espacios públicos como estacionamientos para los automóviles, ya que ello reduce la oferta vial y capacidad de las calles, principalmente por dos razones: resta una pista entera (que es la usada para estacionamiento); y, además, dificulta la circulación de los restantes vehículos cuando uno de ellos efectúa las maniobras para estacionarse.

\section{Conclusiones}

En este trabajo presentamos y discutimos las contribuciones de trabajos empíricos y teóricos con antecedentes relevantes sobre los eventuales efectos adversos que pueden presentarse en la implementación de una política de tarificación vial por congestión. A la luz de estos antecedentes, se puede entregar una explicación de los motivos por los que, en la gran mayoría de las grandes ciudades del mundo donde existen problemas de congestión vehicular, no se ha implementado este tipo de medidas. Los efectos redistributivos que ellas generan, así como el desconocimiento de los beneficios que comportan y del destino de los fondos recaudados, se pueden transformar en obstáculos políticos muy fuertes.

Para impulsar con éxito una política de tarificación vial por congestión, es fundamental considerar al menos tres aspectos relevantes:

a. Los efectos redistributivos que ella puede generar, identificándolos y cuantificándolos.

b. Las expectativas respecto de los reales beneficios que es posible obtener, así como los riesgos latentes, a fin de reducir ambos.

c. Cómo comunicar de manera efectiva los potenciales beneficios atribuibles a una tarificación vial por congestión, así como los destinos de los fondos recaudados. 
El parque automotor aumenta sostenidamente en las distintas ciudades del mundo, haciendo de la congestión un problema creciente. Es esperable que las discusiones sobre esquemas para regular el uso excesivo del automóvil, como la tarificación vial por congestión, sean cada vez más frecuentes. La revisión de las experiencias y el uso de modelos más sofisticados para evaluar los impactos nos ayudan a entender de mejor manera los aspectos relevantes de esta política pública, y así lograr una mayor efectividad en lo que respecta a su diseńo, implementación y posterior evaluación.

\section{Agradecimientos}

Agradecemos el financiamiento del Centro de Desarrollo Urbano Sustentable (cedeus), Conicyt/Fondap/15110020. Rodrigo Troncoso agradece al Proyecto Interfacultades Universidad del Desarrollo.

\section{Referencias bibliográficas}

Ahn, K. \& Rakha, H. (2008). The effects of route choice decisions on vehicle energy consumption and emissions. Transportation Research Part D: Transport and Environment, 13(3), 151167. doi: 10.1016/j.trd.2008.01.005

Anas, A. \& Lindsey, R. (2011). Reducing urban road transportation externalities: Road pricing in theory and in practice, Review of Environmental Economics and Policy, 5(1), 66-88. doi: $10.1093 /$ reep/req019

Anderstig, C., Berglund, S., Eliasson, J., Andersson, M. \& Pyddoke, R. (2012). Congestion charges and labour market imperfections: "Wider economic benefits" or "losses". En Proceeding of the TRB 91th Annual Meeting, Transportation Research Board of the National Academies. Washington D.c. Disponible en http://www.transportportal.se/ SWoPEc/CTS2012-4.pdf

Arnott, R., De Palma, A. \& Lindsey, R. (1993). A structural model of peak-period congestion: A traffic bottleneck with elastic demand. The American Economic Review, 83(1), 161179. En http://www.econ.ucsb.edu/ - tedb/Courses/UCSBpf/readings/arnottetal.pdf

Arnott, R., De Palma, A. \& Lindsey, R. (1994). The welfare effects of congestion tolls with heterogeneous commuters. Journal of Transport Economics and Policy, 28(2), 139-161. En http://www.bath.ac.uk/e-journals/jtep/pdf/Volume_xxvi I I_No_2_139-161.pdf.

Arnott, R., De Palma, A. \& Lindsey, R. (1998). Recent developments in the bottleneck model En K. J. Button \& E. T. Verhoef (Eds.), Road Pricing, Traffic congestion, and the environment (pp. 79-110). Cheltenham, uK: Edward Elgar.

Arnott, R. (2007). Congestion tolling with agglomeration Externalities. Journal of Urban Economics, 62(2), 187-203. doi: 10.1016/j.jue.2007.03.005

Basso, L. J. \& Jara-Díaz, S. R. (2012). Integrating congestion pricing, transit subsidies and mode choice. Transportation Research, 46(6), 890-900. doi: 10.1016/j.tra.2012.02.013

Basso, L. J., Guevara, C. A., Gschwender, A. \& Fuster, M. (2011). Congestion pricing, transit subsidies and dedicated bus lanes: Efficient and practical solutions to congestion. Transport Policy, 18(5), 676-684. doi: 10.1016/j.tranpol.2011.01.002 
Bhatt, K. (1993). Implementing congestion pricing: winners and losers. ITE Journal, 63(12), 33-37.

Beckmann, M., McGuire, C. B. \& C. B. Winsten. (1956). Studies in the economics of transportation. New Haven, CT: Yale University Press.

Benedek, C. M. \& Rilett. L. R. (1998). Equitable traffic assignment with environmental cost function. Journal of Transportation Engineering, 124(1), 16-22. http://dx.doi. org/10.1061/(ASCE)0733-947X(1998)124:1(16)

Bonsall, P. \& Kelly, C. (2005). Road user charging and social exclusion: the impact of congestion charges on at-risk groups. Transport Policy, 12(5), 406-418. doi: 10.1016/j. tranpol.2005.06.007

Cohen, Y. (1987). Commuter welfare under peak-period congestion tolls: Who gains and who loses? International Journal of Transport Economics, 14(3), 239-266.

Correa, J. R., Schulz, A. S. \& Stier-Moses, N. E. (2004). Selfish routing in capacitated networks. Mathematics of Operations Research, 29(4), 961-976. En http://web.mit.edu/schulz/ www/epapers/css-mor-2004.pdf

De Borger, B. \& Proost, S. (Eds.). (2001). Reforming Transport Pricing in the European UnionA Modelling Approach. Cheltenham, uk: Edward Elgar.

De Grange, L. (2010). El gran impacto del Metro. EURE, 36(107), 125-131. En http://www. eure.cl/numero/el-gran-impacto-del-metro/

De Palma, A. \& R. Lindsey. (2011). Traffic congestion pricing methodologies and technologies. Transportation Research, 19(6), 1377-1399. doi: 10.1016/j.trc.2011.02.010

Eliasson, J. \& Mattsson, L. G. (2001). Transport and location effects of road pricing: A simulation approach. Journal of Transport Economics and Policy, 35(3), 417-456. En http://www.ingentaconnect.com/content/lse/jtep/2001/00000035/00000003/ $\operatorname{art} 00005$

Eliasson, J. \& Mattsson, L. G. (2006). Equity effects of congestion pricing: quantitative methodology and a case study for Stockholm. Transportation Research, 40(7), 602620. doi: $10.1016 /$ j.tra.2005.11.002

Evans, A. W. (1992). Road congestion pricing: When is it a good policy? Journal of Transport Economics and Policy, 26(3), 213-243. En http://www.bath.ac.uk/e-journals/jtep/pdf/ Volume_xxvi_No_3_213-243.pdf

Foster, C. (1974). The regressiveness of road pricing. International Journal of Transport Economics, 1(2), 133-141.

Foster, C. (1975). A note on the distributional effects of road pricing: a comment. Journal of Transport Economics and Policy, 9, 186-187.

Franklin, J. P. (2005). The distributional impacts of transportation policies: A Research design for the case of roadway tolls. Documento preparado para la ACSP 46th Annual Conference, oct. 27-30, 2005, Kansas City, MO. En http://www.urbansim.org/pub/ Research/ResearchPapers/franklin-research-design.pdf

Goodwin, P. (1997). Solving congestion: Inaugural lecture of the professorship of transport policy. University College London. En http://www.cts.ucl.ac.uk/tsu/pbginau.htm

Graham, D. J. \& Van Dender, K. (2011). Estimating the agglomeration benefits of transport investments: Some tests for stability. Transportation, 38(3), 409-426. doi: $10.1787 / 5 \mathrm{kmmr} 387 \mathrm{j} 0 \mathrm{zp}$-en 
Glazer, A. \& Niskanen, E. (2000). Which consumers benefit from congestion tolls? Journal of Transport Economics and Policy, 34(1), 43-54. En http://www.bath.ac.uk/e-journals/ jtep/pdf/Volume_34_Part_1_43-53.pdf

Glazer, A. \& Van Dender, K. (2002). How congestion pricing reduces property values? (University of California, Irvine, CA). En http://www.economics.uci.edu/files/economics/docs/ workingpapers/2001-02/Glazer-08.pdf

Hau, T. D. (1992). Economic fundamentals of road pricing. A diagrammatic analysis (World Bank Working Paper 1070). Washington, D.c. The World Bank.

Hau, T. D. (1998). Congestion pricing and road investment. En J. K. Button \& E. Verhoef (Eds.), Road pricing, traffic congestion and the environment: Issues of efficiency and social feasibility (pp. 39-78). Cheltenham, uk: Edward Elgar.

Hau, T. D. (2005). Economic fundamentals of road pricing: A Diagrammatic Analysis, Part I - Fundamentals. Transportmetrica, 1(2), 81-117. doi: 10.1080/18128600508685644

Ison, S. (1998). The saleability of urban road pricing. Economic Affairs, 18(4), 21-25. doi: 10.1111/1468-0270.00122

King, D., Manville, M. \& Shoup, D. (2007). The political calculus of congestion pricing. Transport Policy, 14, 111-123. doi:10.1016/j.tranpol.2006.11.002

Kulash, D. J. (1974). Income-distributional consequences of roadway pricing. Transportation Research Forum Proceedings, 15, pp. 153-59.

Lave, Ch. A. (1995). The demand curve under road pricing and the problem of political feasibility: Authors reply. Transportation Research, 29A, 464-465.

Layard, R. (1977). The distributional effects of congestion taxes. Economica, 44(175), 297-304.

Levinson, D. (2010). Equity effects of road pricing: A review. Transport Reviews, 30(1), 33-57. En http://ssrn.com/abstract $=1735480$

Lindsey, R. (2006). Do economists reach a conclusion on highway pricing?: The intellectual history of an idea. Econ Journal Watch, 3(2), 292-379. En http://1.usa.gov/1APrJpp

Lindsey, R. (2012). Road pricing in a green economy: An unintended consequence or a useful contribution? Theoretical background. Documento presentado en la 46th Annual Pacific Northwest Regional Economic Conference, 18 mayo 2012. En http://www.pnrec. org/wp-content/uploads/2012/05/Lindsay_G1_17.pdf

Liu, L. N. \& McDonald, J. F. (1999). Economic efficiency of second-best congestion pricing schemes in urban highway systems. Transportation Research, 33(3), 157-188. doi: 10.1016/S0191-2615(98)00025-3

Lo, H., Hickman, M. \& Walstad, M. (1996). An evaluation taxonomy for congestion pricing. California PATH Program. Research Report No. UCB-ITS-PRR-96-10 (Berkeley, CA: Institute of Transportation Studies, University of California). En http://www. path.berkeley.edu/sites/default/files/publications/PRR-96-10.pdf

May, A. D. \& Milne, D. S. (2000). Effects of alternative road pricing systems on network performance. Transportation Research, 34(6), 407-436. doi: 10.1016/S09658564(99)00015-4

Mohring, H. \& Harwitz, M. (1962). Highway Benefits: An Analytical Framework. Evanston, IL: Northwestern University Press.

Nash, C. \& Sansom, T. (2001). Pricing European transport systems: Recent developments and evidence from case studies. Journal of Transport Economics and Policy, 35(3), 363-380. En http://eprints.whiterose.ac.uk/2031/ 
Newbery, D. M. (1990). Pricing and congestion: economic principles relevant to road pricing. Oxford Review of Economic Policy, 6(1), 22-38.

Papadimitriou, C. H. (2001). Algorithms, games, and the Internet. Proc. 33rd Annual ACM Sympos. Theory Comput. (STOC), Hersonissos, Greece (pp. 749-753). New York: ACM Press.

Parry, I. W. H. \& Bento, A. (2001). Revenue recycling and the welfare effects of road pricing. Scandinavian Journal of Economics, 103(4), 645-671. doi: 10.1111/1467-9442.00264

Parry, I. W. H. \& Bento, A. (2002). Estimating the welfare effect of congestion taxes: The critical importance of other distortions within the transport system. Journal of Urban Economics, 51(2), 339-365. doi: 10.1006/juec.2001.2248

Parry, I. W. H. (2008). Pricing urban congestion. Resources for the future (Discussion Paper DP-08-35). Washington, D.c.: Resources for the Future. En http://www.rff.org/rff/ Documents/RFF-DP-08-35.pdf

Peters, J. R. \& Kramer, J. K. (2012). Just who should pay for what? Vertical equity, transit subsidy and road pricing: The case of New York City. Journal of Public Transportation, 15(2), 117-136. En http://www.nctr.usf.edu/wp-content/uploads/2012/07/ JPT15.2Peters.pdf

Pigou, A. C. (1920). The economics of welfare. Londres: McMillan.

Raux, C. \& Souche, S. (2004). The acceptability of urban road pricing: A theoretical analysis applied to experience in Lyon. Journal of Transport Economics and Policy, 38(2), 191-216.

Richards, M. (1992). Road pricing: International experience. En Papers Presented at the Congestion Pricing Symposium. June 10-12, organized by the FHWA. FHWA Publication No. FHWA-PL-93-003, 2.1-2.10.

Rilett, L. R. \& Benedek. C. M. (1994). Traffic assignment under environmental and equity objective. Transportation Research Record, 1443, 92-99.

Roughgarden, T. (2003). The price of anarchy is independent of the network topology. Journal of Computer and System Sciences, 67, 341-364. doi: 10.1145/509907.509971

Safirova, E., Houde, S., Coleman, C., Harrington, W. \& Lipman, A. (2006). Long-term consequences of congestion pricing: A small cordon in the hand is worth two in the bush (Discussion Paper DP-06-42). Washington, D.c.: Resources for the Future. En http:// www.rff.org/RFF/Documents/RFF-DP-06-42.pdf

Safirova, E., Harrington, W., Houde, S. \& Coleman, C. (2007). Congestion pricing: myths and realities (Conference Paper 20). Washington, D.c.: Resources for the Future. En http:// www.rff.org/rff/Events/upload/30318_1.pdf

Segal, D. \& Steinmeier (1980). The incidence of congestion and congestion tolls. Journal of Urban Economics, 7(1), pp. 42-62. doi: 10.1016/0094-1190(80)90025-X

Small, K. A. (1983). The incidence of congestion tolls on urban highways. Journal of Urban Economics, 13(1), 90-111. doi: 10.1016/0094-1190(83)90047-5

Small, K. (1992). Urban transportation economics. Newark, NJ: Harwood Academic Publishers.

Small, K. \& Gómez-Ibáńez, J. A. (1998). Road pricing for congestion management: The transition from theory to policyEn J. K. Button \& E. Verhoef (Eds.), Road pricing, traffic congestion and the environment: Issues of efficiency and social feasibility (pp. 213246). Cheltenham, uK: Edward Elgar.

Smith, M. J. (1979). The marginal cost pricing of a transportation network. Transportation Research, 13(3), 237-242. doi: 10.1016/0191-2615(79)90015-8 
Steg, L. \& Schuitema, G. (2007). Behavioural responses to transport pricing: A theoretical analysis. En T. Gärling \& L. Steg (Eds.), Threats to the quality of urban life from car traffic: Problems, causes, and solutions (pp. 347-366). Amsterdam: Elsevier.

Sugawara, S. \& Niemeyer, D. A. (2002). How much can vehicle emissions be reduced? Exploratory analysis of an upper boundary using an emission-optimized trip assignment. Transportation Research Record, 1815(1), 29-37. doi: 10.3141/1815-04

Tsekeris, T. \& Voß, S. (2009). Design and evaluation of road pricing: State-of-the-art and methodological advances. Netnomics: Economic Research \& Electronic Networking, 10(1), 5-52. doi 10.1007/s11066-008-9024-z

Tzeng, G. H. \& Chen, C. H. (1993). Multiobjective decision-making for traffic assignment. IEEE Transactions on Engineering Management, 40(2), 180-187. doi: http://dx.doi. org/10.1109/17.277411

Verhoef, E. T., Nijkamp, P. \& Rietveld, P. (1996). Second-best congestion pricing: the case of an untolled alternative. Journal of Urban Economics, 40(3), 279-302. doi: 10.1006/ juec. 1996.0033

Verhoef, E. T. \& Small, K. A. (2004). Product differentiation on roads: constrained congestion pricing with heterogeneous users. Journal of Transport Economics and Policy, 38(1), 127-156. En http://www.uctc.net/papers/656.pdf

Vickrey, W. (1963). Pricing in urban and suburban transport. American Economic Review: Paper and Proceedings, 53(2), 452-465.

Walters, A. (1961). The theory and measurement of marginal private and social costs of highway congestion. Econometrica, 29, 676-699.

Wilson, P. W. (1988). Welfare effects of congestion pricing in Singapore. Transportation, 15(3), 191-210. http://dx.doi.org/10.1007/BF00837581

Yang, H., Xu, W. \& Heydecker, B. (2010). Bounding the efficiency of road pricing. Transportation Research, 46(1), 90-108. doi: 10.1016/j.tre.2009.05.007

Yin, Y. \& Lawphongpanich, S. (2006). Internalizing emission externality on road networks. Transportation Research Part D: Transport and Environment, 11(4), 292-301. doi: 10.1016/j.trd.2006.05.003 